\title{
Solving Nesting Problems with Non-Convex Polygons by Constraint Logic Programming *
}

\author{
Maria Antónia Carravilla ${ }^{\dagger \ddagger} \quad$ Cristina Ribeiro ${ }^{\dagger \ddagger}$ \\ José F. Oliveira ${ }^{\dagger \ddagger}$ \\ Rua Dr. Roberto Frias s/n, 4250-465 Porto, Portugal \\ $\{$ mac,mcr,jfo\}@fe.up.pt
}

\begin{abstract}
In this paper an application of constraint logic programming (CLP) to the resolution of nesting problems is presented. Nesting problems are a special case of the cutting and packing problems, in which the pieces generally have non-convex shapes. Due to their combinatorial optimization nature, nesting problems have traditionally been tackled by heuristics and in the recent past by meta-heuristics. When trying to formulate nesting problems as linear programming models, to achieve global optimal solutions, the difficulty of dealing with the disjunction of constraints arises. On the contrary, CLP deals easily with this type of relationships among constraints. A CLP implementation for the nesting problem is described for convex and non-convex shapes. The concept of no-fit polygon is used to deal with the geometric constraints inherent to all cutting and packing problems. Computational results are presented.
\end{abstract}

Keywords: packing, nesting, artificial intelligence, constraint logic programming

\section{Introduction}

In the cutting and packing problems one or more pieces of material or space must be divided into smaller pieces. The minimisation of the waste is usually the main objective of these combinatorial optimisation problems. Figure 1 presents examples of cutting and packing problems of different dimensions.

In the nesting problems only two dimensions, the length and the width, are relevant since the other dimension is common to all the pieces. Moreover

\footnotetext{
*Partially supported by FCT, POSI and FEDER (POSI/33757/SRI/2000 (CLPnest))

${ }^{\dagger}$ Faculty of Engineering of the University of Porto

${ }^{\ddagger}$ Institute of Computers and Systems Engineering - Porto
} 


\section{Dimensional Cutting}

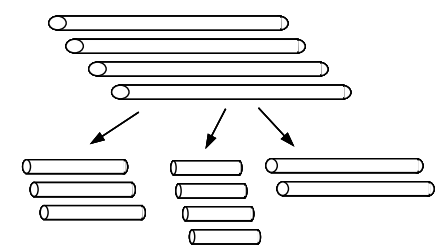

2 Dimensional Rectangular Cutting

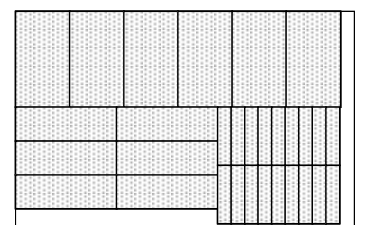

Pallet Loading

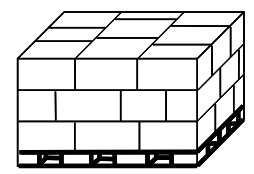

2 Dimensional Nesting Problem

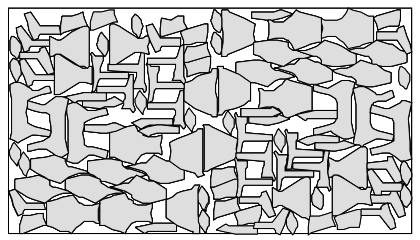

Figure 1: Examples of cutting and packing problems

the small pieces have an irregular (non-rectangular) shape and are generally non-convex. The variant of the problem that we are going to deal with considers only one big piece, the plate, with fixed width and infinite length. A given set of small pieces must be placed over the plate while trying to minimise the total length used. Both convex and non-convex pieces will be considered in this work. A more detailed description of nesting problems and cutting and packing problems can be found in [DD92, DD95].

Nesting problems have traditionally been tackled mainly by heuristic algorithms, that try to generate good solutions following specific strategies when searching the solution space. In this context several approaches, ranging from simple heuristics to local optimisation techniques (including, more recently, meta-heuristics) [Art66, AS80, DDB98, OF93, BHW93, MD, SY98] have been proposed. However none of them has been able to generate global optimal solutions.

The other traditional way of tackling combinatorial optimization problems, building mixed integer programming models and solving them with appropriate software, allows only the resolution of Nesting Problems of very small size. Even for small instances of the problem and considering only convex shapes, the mathematical programming model does not generate global optimal solutions in a reasonable amount of time.

A more flexible approach is proposed in this paper using Constraint Logic Programming (CLP). CLP provides a very convenient framework for this class of problems. An acceptable cutting pattern is naturally expressed via the constraints imposed on each pair of pieces therein. The minimisation criterion, on the other hand, is easily turned into a search strategy.

In practice, in combinatorial problems there is much more knowledge 
about the nature of the problem than what can be put into the constraints that define a solution. CLP provides the means to express this knowledge in a flexible way, and so, all the additional features of the problem - extra constraints, redundant constraints, symmetries, partial solutions which are not viable and should be abandoned - can be accounted for in an expressive way. The main advantage of a CLP formulation for the problems in this class is that it does not need to conform to a rigid model. It lends itself very naturally to the integration of the intrinsic features of the problem and to the use of heuristic methods for obtaining initial solutions.

In [RCO99a] a CLP approach to the resolution of nesting problems is presented, considering only convex shapes. In [RCO99b], some ideas for handling non-convex polygons have been introduced. The focus of this paper is on the development and application of these ideas to the resolution of nonconvex nesting problems.

In the next section the basic concept to satisfy the geometric constraints between poligonal pieces, the nofit polygon, is introduced. Then the main concepts of Constraint Logic Programming are presented, followed by the description of its application to nesting problems. Finally some experiments and results will be presented and discussed.

\section{The nofit polygon}

In nesting problems the combinatorial problem coexists with a geometric problem, since solutions must be "geometrically" feasible: pieces may not overlap and must completely fit inside the plate. In the approach described in this paper the geometric constraints of the problem have been tackled with the nofit polygon.

The concept of nofit polygon was first introduced by Art [Art66] and was used again by Adamowicz and Albano [AA72, AA76]. Later Mahadevan [Mah84] has presented a comprehensive description of an algorithm to build the nofit polygon.

The nofit polygon of piece $B$ relative to piece $A\left(N F P_{A B}\right)$ is the locus of points traced by the reference point of $B$, when it slides along the contour of $A$. The relative orientations of $A$ and $B$ are maintained during this orbital movement. Piece $B$ (the orbital piece) must never intersect piece $A$ (the stationary piece) and they must always be in contact (see Figure 2).

From this definition it immediately follows that:

- If the reference point of piece $B$ is placed in the interior of $N F P_{A B}$ then $B$ intersects $A$.

- If the reference point of piece $B$ is placed on the boundary of $N F P_{A B}$ then $B$ touches $A$. 


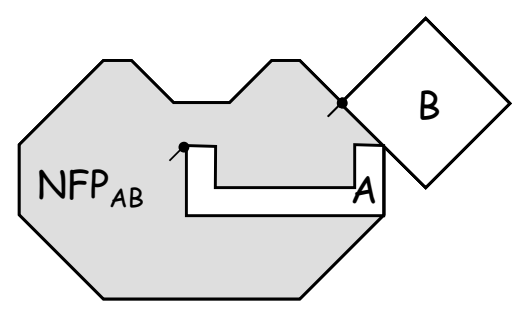

Figure 2: The nofit polygon of piece $B$ relative to piece $A$

- If the reference point of piece $B$ is placed in the exterior of $N F P_{A B}$ then $B$ does not intersect or touch $A$.

To achieve a feasible (non-overlapping) and tight layout, each piece should have its reference point on the boundary of at least one nofit polygon relative to another piece and in the exterior of the nofit polygons relative to all the other pieces.

The combinatorial problem arises when choosing a placement point for each piece, among its set of feasible placement points, knowing that the choice made will restrict the feasible set of placement points of all the other pieces.

\section{Constraint programming}

\subsection{Constraints in OR and programming languages}

The mathematical models used in Operational Research resort to the concept of constraint for their formulation. The combinatorial nature of some families of problems arises from the fact that some variables used to model them must take values in a discrete domain, subject to a set of constraints. Constraints in Operational Research are used in a very disciplined way, always having to conform to predefined families of models.

Programming languages, on the other hand, have evolved in the sense of improving the abstractions available to the programmer, allowing procedures and data structures to more directly match the functions and entities in the application domain. Up to the advent of Constraint Programming, programming languages lacked support for handling constraints among programdefined objects. The burden of handling the constraints intrinsic to a problem domain was therefore left to the programmer.

Traditionally there have been two approaches to solve combinatorial problems in Operational Research: build a standard model and apply it to a specialized solver or develop a customized programme in a traditional programming language. The former takes advantage of the specialized algorithms built into the solver, but forces the user to cast the problem into 
Conventional Programme

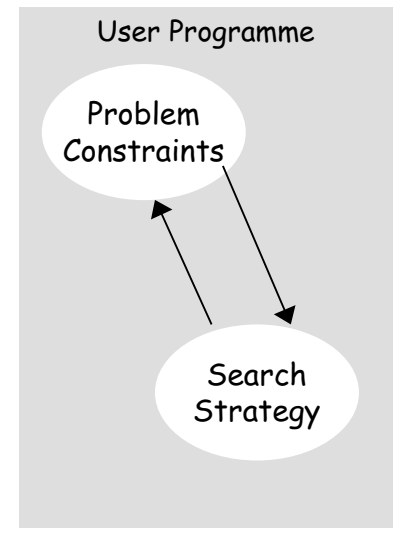

CLP Programme

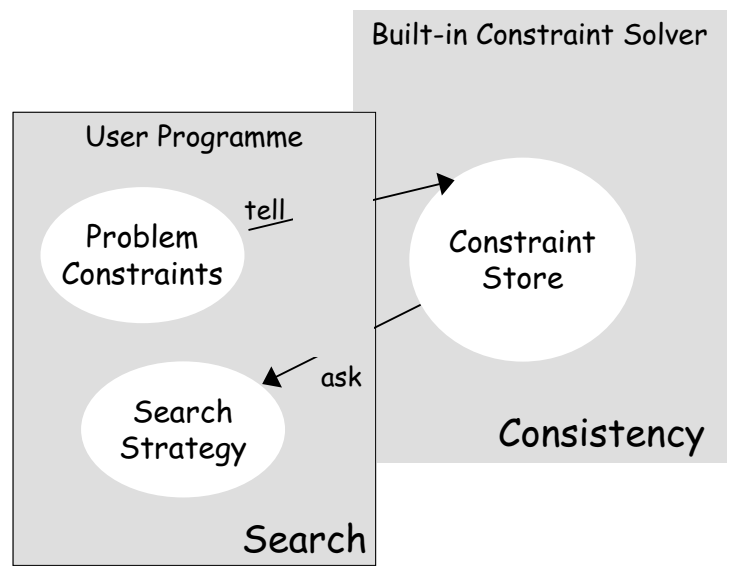

Figure 3: Single-layer programme versus dual-layer CLP model

a rigid model. The latter allows the customization of an application to the features of a problem category but requires a large investment on the coding of algorithms for efficient constraint handling.

\subsection{Constraint programming in OR}

Constraint programming languages are regular general-purpose programming languages with specialised operations to deal with constraints. From an Operational Research perspective, a constraint programming language is used instead of a modelling language [MS98, Tsa93]. It has the advantage of allowing the statement of more generic constraints and offers the possibility of developing specific problem-solving strategies, while making use of the built-in constraint solving algorithms. ¿From the programming language point of view, constraint solving is added as a second layer in the execution environment. Figure 3 illustrates the difference between a conventional single-layer programme for a search problem, where constraints have to be solved by the user programme, and the dual-layer model offered by constraint programming.

The problem with a dual-layer model is how to efficiently interleave constraint handling with the adopted problem solving strategy. In constraint programming, the answer is to actively use the constraints for reducing the domains of the variables and to use different constraint handling algorithms depending on the type of constraints. 


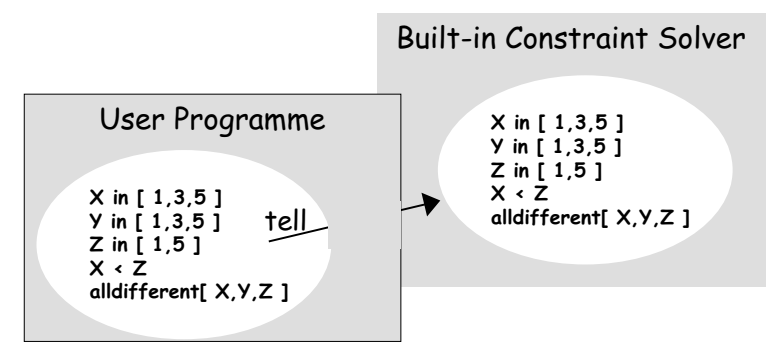

Figure 4: Constraints are added to the constraint store

\subsection{Example}

Consider the example in Figure 4 where we have 3 variables: $X$ and $Y$ that may take one of 3 values, 1,3 or 5 and $Z$ that may take one of two values, 1 or 5 . There are two constraints on the variables. The first is

$$
X<Z
$$

and the second is that the three variables must take different values.

In a constraint programming language the first constraint is a typical primitive constraint and the second is also available as a constraint manipulated by a specialized algorithm. For this purpose we will be using the constraint

$$
\text { alldifferent }(X, Y, Z)
$$

Both the domains of the variables and the constraints are added to the constraint store.

Adding constraints originates their so-called propagation, removing infeasible values from their domains. The first propagation step uses the first constraint and removes 5 from $X$ 's domain and 1 from $Z$ 's domain, as shown on Figure 5. The constraint $X<Z$ has become entailed due to the current state of the variables and therefore will not be considered again.

With the new domains, the second constraint is activated and the value 5 is removed from $Y$ 's domain, see Figure 6.

There is no further propagation and the search for solutions has to proceed by exploring the values in the domains of the variables. The key point here is that, as the search for solutions proceeds, constraint propagation is activated each time the value of a variable changes.

Assuming a search strategy that chooses the variable with the smaller domain first, for $Z=5, X$ may take values 1 or 3 . Choosing the value 3 first, a propagation step occurs and 3 is removed from $Y$ 's domain, see Figure 7.

The solution $X=3, Y=1, Z=5$ is found. Backtracking on the available choice gives $X=1, Y=3, Z=5$. 


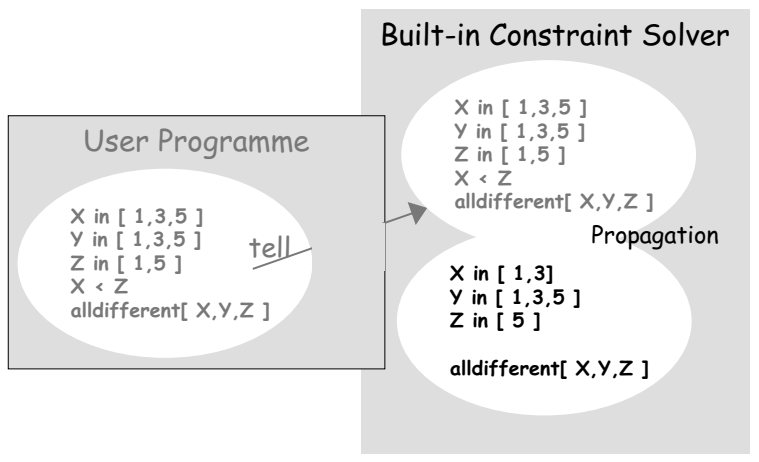

Figure 5: Propagation - first step

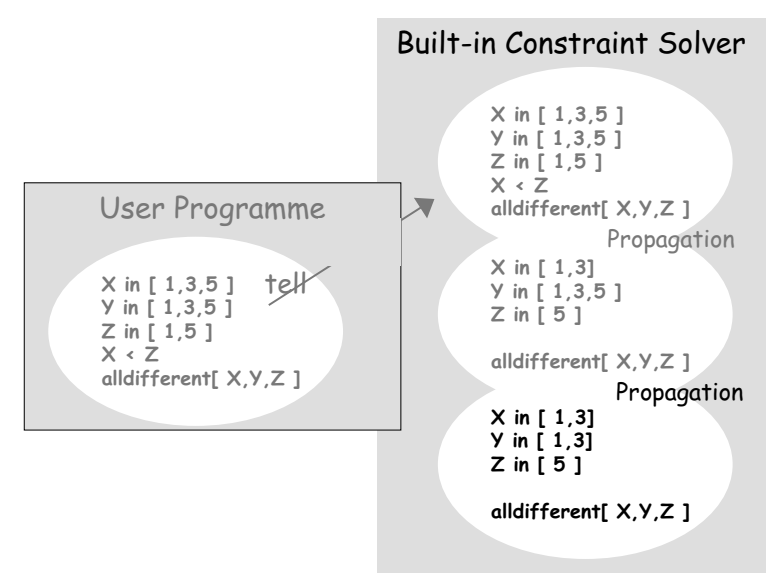

Figure 6: Propagation - second step

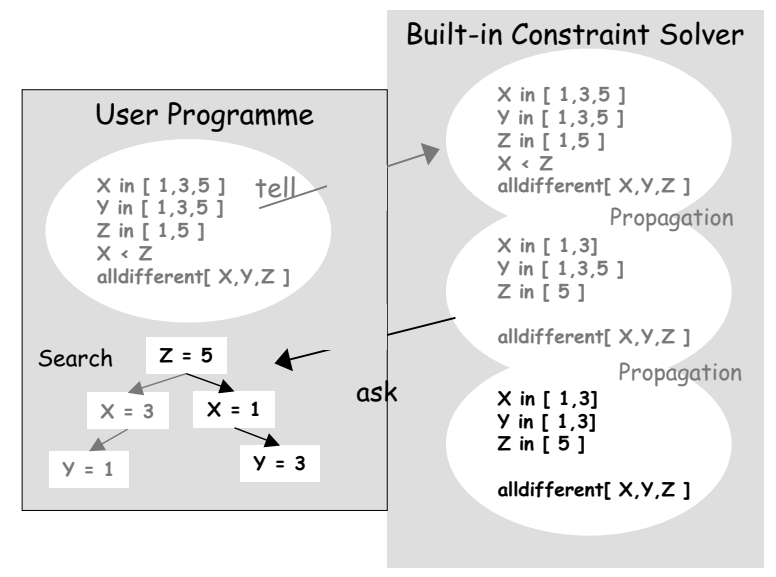

Figure 7: Search and propagation 
This is obviously a very simplistic example, based on finite domain variables, domain consistency and sequential exploration of the domains. The specification of constraints in CP languages typically allows variants in the propagation of constraints and offers other methods for exploring the domains.

\subsection{Constraint programming in CLP}

Logic Programming favours programming in a declarative style: a programme states what the solution of a problem is and the computational mechanism determines how a specific solution is obtained. The resolution of combinatorial problems expressed with constraints always leads to the exploration of a solution space. In logic programming languages the concept of backtrack on a partial solution is basic and the computation state is efficiently maintained at low level. This is therefore a convenient paradigm for search problems.

Constraint Logic Programming (CLP) appeared as the extension of logic programming in order to allow the constraints on variables to be more than just the equality constraints expressed by variable bindings [JM94]. The extension is based on the existence of a constraint solver as a specialised engine used to store, reduce and in general enforce the constraints on selected variables. Enforcing consistency is the main feature of a built-in constraint solver. A computation branch that generates an inconsistency between constrained variables is abandoned.

Constraint programming, however, is not restricted to constraint logic programming. Constraint manipulation has been added as a built-in resource in languages that follow the object-oriented paradigm; an example is Ilog-Solver [ILO01] where both constraint handling and search are offered as extensions to $\mathrm{C}++$.

The prototype applications described here use CLP-FD of Sicstus Prolog [Swe95, COC97], which handles constraints on finite domains. Although the nesting problems are not intrinsically finite domain problems, it is common to solve them with some predefined granularity. This is also convenient for benchmarking and comparison, as many state-of-the-art approaches to the solution of nesting problems adopt discrete models for the positioning space.

\section{Applying CLP to nesting}

The input data in a nesting problem are the width and an upper bound on the length of the plate, together with the description of the small pieces (polygons). The output data are the position of the placement points $(X, Y)$ of all the pieces. The objective is to determine all these positions, such that the pieces do not overlap and the length of the plate used to place them is minimised (Figure 8). 


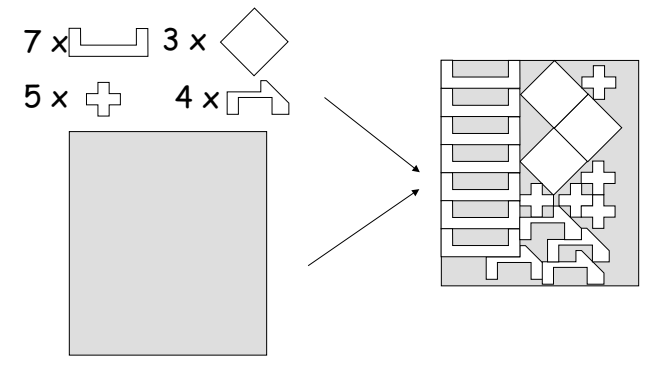

Figure 8: Input and output data of a nesting problem

\subsection{Initial domains}

Given the input data, the initial domains of the placement point of each polygon need to be computed (Figure 9). By inspecting Figure 9, one can verify that if the placement point $\left(X_{j}, Y_{j}\right)$ is outside the white area or it's boundaries, then piece $j$ is not totally inside the plate.

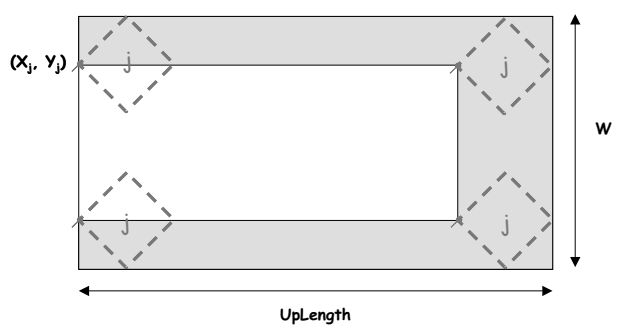

Figure 9: Polygon $j$ : initial domains of the placement point $\left(X_{j}, Y_{j}\right)$

The constraint that must be satisfied is that the polygon must fit inside the plate. Given the width $W$ and an upper bound on the length of the plate (UpLength), the initial domain for each placement point is obtained using the rectangular envelope of the piece $\left(X_{j \min }, Y_{j \min }, X_{j \max }, Y_{j \max }\right)$.

$$
\text { doDomains }\left(W, U \text { plength }, X_{j \min }, Y_{j \min }, X_{\text {jmax }}, Y_{\text {jmax }}, X_{j}, Y_{j}\right)
$$

\subsection{Constraints}

The geometric constraints of the nesting problem are that the pieces should not overlap. These constraints were built using the nofit polygons (NFP) of each pair of pieces. Piece $j$ does not overlap piece $i$ if its placement point is outside or on the boundary of the $N F P_{i j}$.

In the case of convex NFP's, this is equivalent to stating that the placement point must be over or on the left hand side of at least one of the edges 

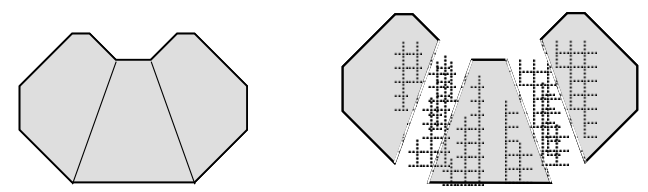

Figure 10: Decomposition of a non-convex polygon

of the $N F P_{i j}$, assuming a clockwise path. The fact that a point is to the left of an edge is expressed using the D-functions as described in [Mah84].

Non-convex NFP's can be decomposed into convex sub-polygons. This process will however generate "fake" edges which lay in the interior of the original NFP and therefore do not represent feasible positioning points (see Figure 10). The statement that the placement point of a piece is outside or on the boundary of the $N F P$ is equivalent to the statement that the placement point must be over or on the left-hand side of at least one of the edges of all its sub-polygons. Additionally, the placement point can never be over the edges which are inside the $N F P$, as they only appear as a consequence of polygon decomposition.

$$
\text { doConstraintsNFP }\left(X_{i}, Y_{i}, X_{j}, Y_{j}, N F P_{i j}\right)
$$

Assuming that piece $i$ is already placed at point $\left(X_{i}, Y_{i}\right)$, the reduced domains for the placement point of piece $j,\left(X_{j}, Y_{j}\right)$ are represented in Figure 11. The white area and its boundaries represent possible positioning points for piece $j$.

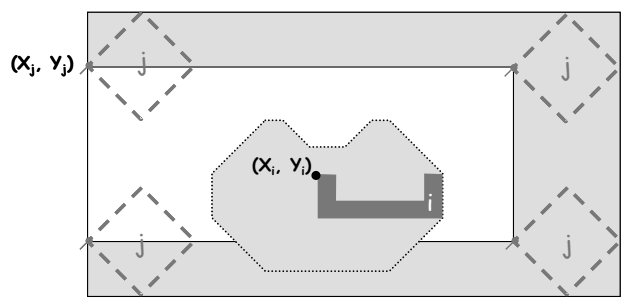

Figure 11: Domains of $\left(X_{j}, Y_{j}\right)$, if $\left(X_{i}, Y_{i}\right)$ are fixed

Note that the reduction of the domain for $\left(X_{j}, Y_{j}\right)$ as a consequence of the positioning of piece $i$, does not have to be explicitly programmed. The binding of the positioning point of piece $i$ to a single point automatically generates the propagation of constraints in the constraint store. As a result, the domains of the positioning points for all the other pieces are accordingly reduced.

For identical pieces (pieces with the same shape), symmetric positionings are avoided (see Figure 12). This is accomplished by imposing additional 

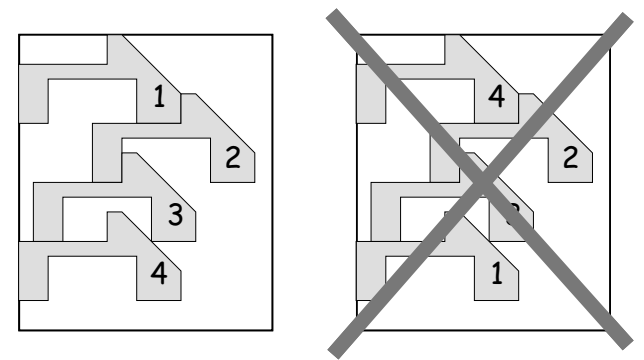

Figure 12: Symmetry constraints

constraints on their $X$ and $Y$ coordinates. Identical pieces are assigned in an 'a priori' arbitrary order. For identical pieces $k$ and $p$ such that piece $k$ precedes piece $p$, the constraint $X_{p} \leq X_{k}$ is imposed. In case $X_{k}=X_{p}$, then the constraint $Y_{k} \leq Y_{p}$ is imposed.

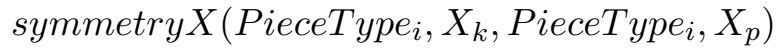

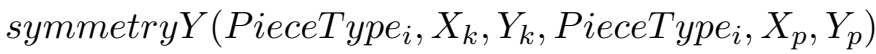

\subsection{CLP resolution strategy}

The generation of solutions, in the form of positioning patterns, has followed a standard CLP resolution strategy using finite-domain variables. Each piece is represented by a pair of variables, the $x$ and $y$ coordinates of its positioning point. The geometric constraints are then imposed on each pair of pieces. Enumeration is used in the final stage to search the solution space.

The initial domains are built using the width and the upper bound of the length of the plate, and the geometry of the small pieces. The nofit polygons are used in the generation of the constraints on the placement points for all pairs of small pieces. For identical pieces, symmetric positionings are avoided. The constraint solver keeps track of changes in the domains of variables. When the position of one piece is fixed, the domains of the positioning points of all the other pieces are automatically reduced. Figure 13 illustrates the integration of the constraint solver with the search module.

On the enumeration phase the choice of values for the positioning points follows the 'a priori' ordering of the small pieces. For any two acceptable positions for the same piece, the leftmost is preferred. Along the search, the best solution found so far is memorised, so that, for every single piece, a position beyond the best solution obtained is not considered. 


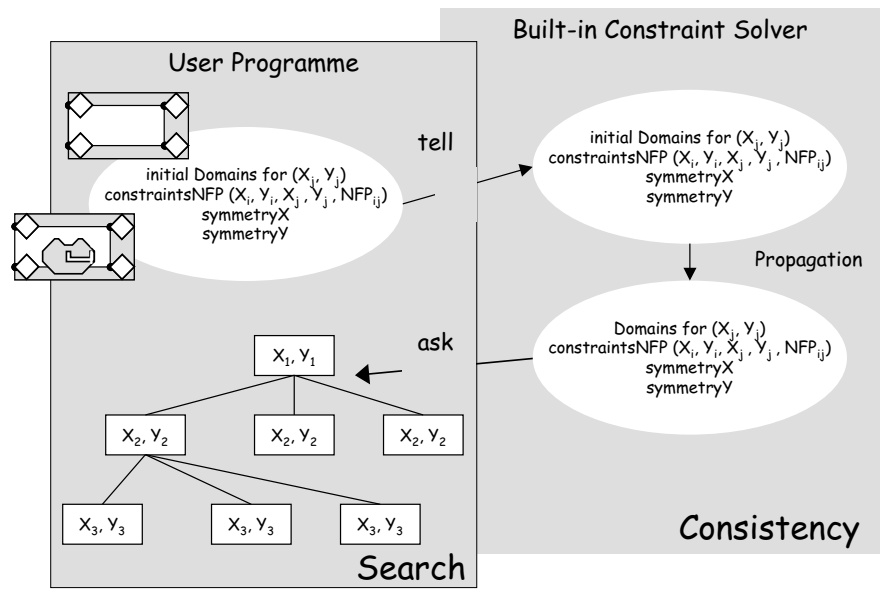

Figure 13: Integration of constraint solving and search

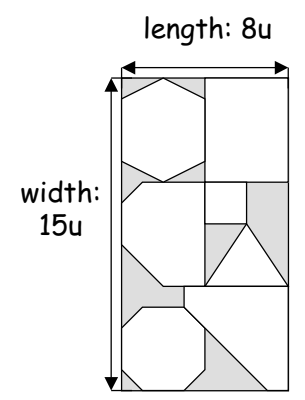

Figure 14: Optimal solution for example $R C O 7$

\section{Some results}

The tests were made using SICStus-Prolog CLP-FD module (version 3.8.5) ([Swe95]) on a $733 \mathrm{MHz}$ Pentium with $256 \mathrm{Mb}$ Ram. The approach described here was tested on two examples, one having only convex polygons $(R C O)$ and another having both convex and non-convex polygons (shapes0).

$R C O 7$ uses a set of seven convex pieces and a plate width of 15 length units. It took approximately 250 milliseconds to find the solution shown in Figure 14, and about 22 seconds to prove its optimality. The Mixed Integer Programming model for this problem would have 14 continuous variables, 179 binary variables and 235 constraints. The number of CLP-constraints for this problem is 179 .

$R C O 35$ uses a set of $5 \times 7$ convex polygons. It took about 5 seconds to find a solution with length 38 , and around 20 minutes to obtain the solution (length 37) shown in Figure 15. The Mixed Integer Programming model for 


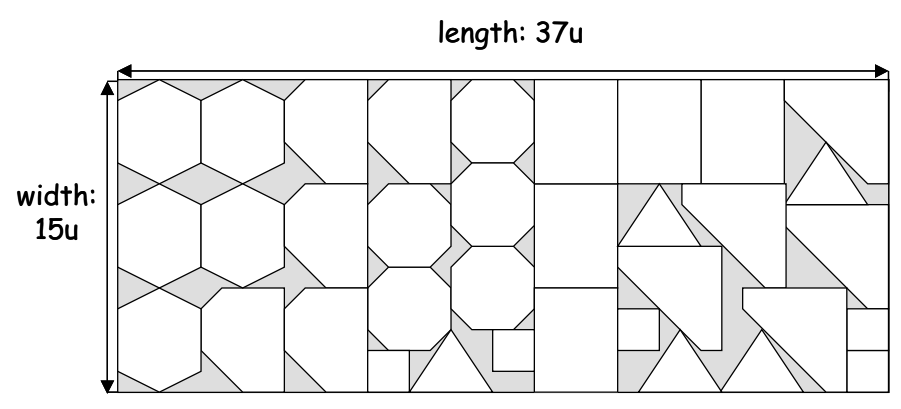

Figure 15: Solution for example $R C O 35$

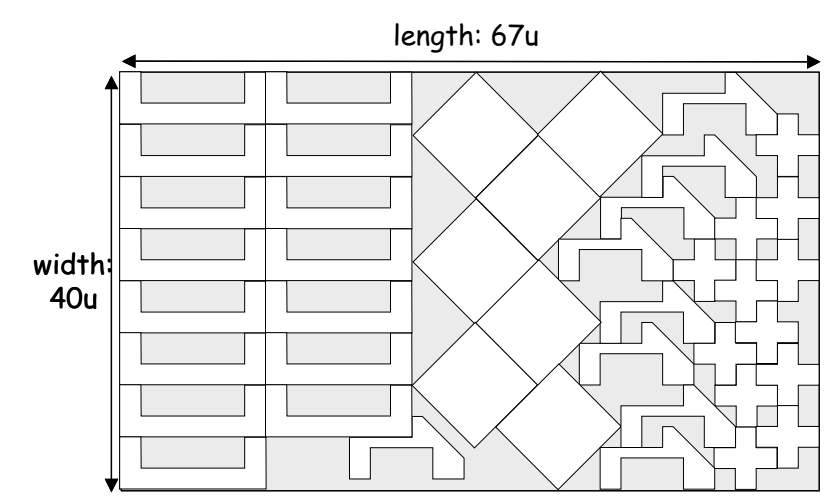

Figure 16: Solution for shapes0 with length 67 (obtained in 55 seconds)

this problem would have 4400 binary variables, 70 continuous variables and 5100 constraints. The number of CLP-constraints for this problem is 5245 .

shapes 0 uses a set of 43 non-convex and convex polygons and the number of CLP-constraints for this problem is 32961. The best solution obtained for the instance shapes 0 is presented in Figure 16. The best solution known in the literature for the problem shapes 0 , using integer positioning points has a length of 63 [DD95].

\section{Conclusions and further research}

In this paper, a first approach to the use of CLP in the resolution of general nesting problems was presented. The CLP framework provided a very manageable expression of the nesting problems where it has been easy to add constraints and to specialise the search methods according to all the knowledge that exists on the features of the problem.

Future research will explore CLP in the real domain as opposed to the integer domain of CLP-FD. It is also possible to incorporate heuristic rules in 
the search, specially for large-scale problems where one is satisfied with good solutions obtained in a reasonable amount of time. The introduction of more redundant constraints can activate further domain reduction, simplifying the search. Finally, the integration of heuristic methods with CLP is being explored; CLP can be used to provide initial solutions or to explore parts of the search space.

\section{References}

[AA72] M Adamowicz and A. Albano. A two-stage solution of the cutting-stock problem. Information Processing, 71:1086-1091, 1972 .

[AA76] M Adamowicz and A. Albano. A solution of the rectangular cutting-stock problem. IEEE Transactions on Systems, Man and Cybernetics, 6(4):302-310, 1976.

[Art66] R.C. Art. An approach to the two-dimensional, irregular cutting stock problem. Technical Report 36.008, IBM Cambridge Centre, 1966.

[AS80] A. Albano and G. Sapuppo. Optimal allocation of twodimensional irregular shapes using heuristic search methods. IEEE Transactions on Systems, Man and Cybernetics, SMC10(5):242-248, 1980.

[BHW93] J. Błażewicz, P. Hawryluk, and R. Walkowiak. Using tabu search approach for solving the two-dimensional irregular cutting problem in tabu search. In F. Glover, M. Laguna, E. Taillard, and D. de Werra, editors, Tabu Search, volume 41 of Annals of Operations Research. J. C. Baltzer AG, 1993.

[COC97] Mats Carlsson, Greger Ottosson, and Björn Carlson. An openended finite domain constraint solver. In H. Glaser, P. Hartel, and H. Kucken, editors, Programming Languages: Implementations, Logics, and Programming, volume 1292 of Lecture Notes in Computer Science, pages 191-206, Southampton, September 1997. Springer-Verlag.

[DD92] K. Dowsland and W. Dowsland. Packing problems. European Journal of Operational Research, 56:2-14, 1992.

[DD95] K. Dowsland and W. Dowsland. Solution approaches to irregular nesting problems. European Journal of Operational Research, 84:506-521, 1995. 
[DDB98] K.A. Dowsland, W.B. Dowsland, and J.A. Bennell. Jostling for position: Local improvement for irregular cutting patterns. Journal of the Operational Research Society, 49:647-658, 1998.

[ILO01] ILOG. "ILOG Solver 5.1 User's Manual", 2001.

[JM94] Joxan Jaffar and Michael J. Maher. Constraint logic programming: A survey. Journal of Logic Programming, 19/20:503-581, 1994.

[Mah84] A. Mahadevan. Optimization in Computer-Aided Pattern Packing. PhD thesis, North Carolina State University, 1984.

[MD] V. Milenkovic and K. Daniels. Translational polygon containment and minimal enclosure using mathematical programming. Submited to the International Transactions in Operational Research.

[MS98] Kim Marriott and Peter J. Stuckey. Programming with Constraints - an Introduction. MIT Press, 1998.

[OF93] J.F. Oliveira and J.S. Ferreira. Algorithms for nesting problems. In René V. V. Vidal, editor, Applied Simulated Annealing, Lecture Notes in Economics and Mathematical Systems, pages 255273. Springer-Verlag, 1993.

[RCO99a] Cristina Ribeiro, Maria Antónia Carravilla, and José Fernando Oliveira. Applying constraint logic programming to the resolution of nesting problems. Pesquisa Operacional, 19(2):239-247, 1999.

[RCO99b] Cristina Ribeiro, Maria Antónia Carravilla, and José Fernando Oliveira. Applying constraint logic programming to the resolution of nesting problems. In Workshop on Integration of AI and OR techniques in Constraint Programming for Combinatorial Optimization Problems, 1999.

[Swe95] Swedish Institute of Computer Science. SICStus Prolog User's Manual, 1995.

[SY98] Y.G. Stoyan and G.N. Yaskov. Mathematical model and solution method of optimization problem of placement of rectangles and circles taking into account special constraints. International Transactions on Operational Research, 5(1):45-57, January 1998.

[Tsa93] Edward Tsang. Foundations of Constraint Satisfaction. Academic Press, 1993. 